\title{
Stability Constraints for Robust Model Predictive Control
}

\author{
Amanda G. S. Ottoni, ${ }^{1}$ Ricardo H. C. Takahashi, ${ }^{2}$ and Guilherme V. Raffo ${ }^{3}$ \\ ${ }^{1}$ Department of Physics and Mathematics, Federal University of São João del-Rei, Campus Alto Paraopeba, Rodovia MG 443, \\ KM 7, 36420-000 Ouro Branco, MG, Brazil \\ ${ }^{2}$ Department of Mathematics, Federal University of Minas Gerais, Campus da Pampulha, Avenida Antônio Carlos 6627, \\ 31270-901 Belo Horizonte, MG, Brazil \\ ${ }^{3}$ Department of Electronic Engineering, Federal University of Minas Gerais, Campus da Pampulha, Avenida Antônio Carlos 6627, \\ 31270-901 Belo Horizonte, MG, Brazil \\ Correspondence should be addressed to Ricardo H. C. Takahashi; taka@mat.ufmg.br
}

Received 18 August 2015; Revised 15 October 2015; Accepted 27 October 2015

Academic Editor: Yan-Jun Liu

Copyright (C) 2015 Amanda G. S. Ottoni et al. This is an open access article distributed under the Creative Commons Attribution License, which permits unrestricted use, distribution, and reproduction in any medium, provided the original work is properly cited.

\begin{abstract}
This paper proposes an approach for the robust stabilization of systems controlled by MPC strategies. Uncertain SISO linear systems with box-bounded parametric uncertainties are considered. The proposed approach delivers some constraints on the control inputs which impose sufficient conditions for the convergence of the system output. These stability constraints can be included in the set of constraints dealt with by existing MPC design strategies, in this way leading to the "robustification" of the MPC.
\end{abstract}

\section{Introduction}

The term Model Predictive Control, MPC, stands for a wide range of control methods which make an explicit usage of the process model to obtain the control signal by minimizing an objective function [1]. It is difficult to determine the exact origin of MPC techniques, but it is known that they were developed in the 70 s to solve control problems related to chemical industry and oil refining [2]. Currently, their application has been widespread in other sectors. The main features that have contributed to the growing use of predictive controllers, according to Maciejowski [3], are their ability to deal with time delays. Physical and operational constraints can be incorporated directly in the control design, reducing the number of emergency stops of the system. This makes the MPC controllers efficient and able to operate for long periods without requiring intervention [4].

A critical step in the design of MPC controllers is the determination of plant model to be used in the prediction. Discrepancies between the plant and its model may lead to poor performances or even to system instability. This situation motivated the development of robust MPC techniques that are intended to preserve stability and performance, despite inaccuracies or uncertainties in the model. As pointed out by Mayne [5], "while major aspects of nominal MPC were well understood by 2000, the presence of uncertainty (...) and the associated topic of robustness against uncertainty, is a major challenge that is still receiving considerable attention." According to Mayne, assuming that the decision variable is a control sequence, there are three general approaches that are followed in MPC design for uncertain systems: (i) to take the uncertainty into account by requiring that the control problem constraints are satisfied for all possible realizations of the disturbance sequence; (ii) to employ a local feedback around a nominal trajectory; and (iii) to consider unstructured uncertainty in the system model.

Under the approach (i), the earlier literature employed the nominal value of the objective function of the MPC as a Lyapunov function [6-8]. In [9] a robust invariant terminal set was employed in order to ensure recursive feasibility. Several papers [10-12] addressed the problem of min-max MPC, in which the objective function is defined as the maximum, over the uncertainty set, of a cost function. Such a methodology is theoretically interesting, but it tends to be computationally costly. Reference [13] proposed an approximated min-max approach in order to reduce the 
computational burden. More recently, the robust stability of MPC has been studied under the viewpoint of the input-tostate stability (ISS) [14, 15].

The approach (ii), also called the tube-based MPC, employs simple parameterized local policies in order to approximate the (ideal) optimization over the control policies, instead of performing the optimization over the control sequences. References [16-18] follow this approach.

Finally, the approach (iii) usually relies on the small gain theorem. The first attempt to follow this direction was developed in the works [19-21], employing a frequency domain approach. In [22], the standard $\mathscr{H}_{\infty}$ model is employed. A related approach is described in [23].

It is also worth mentioning MPC schemes for nonlinear systems. For instance, [24] presents a methodology which is based on an adaptive neural network. In [25], a neural network is also employed in order to achieve the robustness of the MPC against uncertainties in the control input matrix. The paper [26] performs an MPC that deals with nonlinear systems with dead-zone input. Other related references are $[27,28]$.

This paper proposes a methodology for the robust stabilization of uncertain SISO systems with parametric uncertainty which can be situated in the approach (i) above. The issue of stabilization is stated here from its first principles, as a feasibility problem related to the convergence of a sequence. This stability condition can be treated as a constraint in the MPC synthesis, taking advantage of the easy management of constraints within MPC. This defines a problem of optimal control synthesis with a constraint of robust stability which, itself, is calculated as a solution of a nonlinear optimization problem. It should be noticed that the algorithm for solving such a problem involves the solution of a subproblem of nonlinear optimization within an algorithm step which requires some computation time that may prevent the application of the proposed scheme to systems with small sampling times. In relation to this issue, the following points should be mentioned: (i) Several important plants have sampling times that are within the range of several minutes to several hours. In those cases, it is better to apply a more precise and less conservative algorithm that runs in some seconds than an approximate algorithm that runs in some milliseconds. (ii) Any proposal of robust MPC that adopts approximated formulae and conservative bounds in order to achieve fast computation should be benchmarked against a less conservative version of the same strategy. The proposed approach can be used as a benchmark for the performance of other MPC design procedures.

The proposed methodology is compared with the design technique of class (i) presented in [13] and with the design technique of class (iii) presented in [19-21]. Computational experiments show that the proposed methodology leads to the stabilization of plants belonging to larger uncertainty sets, considering parametric uncertainties represented by interval sets. The results suggest that the proposed methodology can be less conservative in the case of uncertainty sets of that type.

The remainder of this paper is structured as follows. Section 2 presents the problem statement. Section 3 describes the proposed formulation. Section 4 shows simulation results. Section 5 closes the paper with some conclusions.

\section{Problem Statement}

Consider a system described by a CARIMA (Controlled AutoRegressive Integrating Moving-Average) model, as follows [1]:

$$
\Delta y(k)=T_{\mathbf{a}, \mathbf{b}}(z) \Delta u(k)
$$

where the term $\Delta=1-z^{-1}$ corresponds to an integral action that enables deleting the static error and

$$
T_{\mathbf{a}, \mathbf{b}}(z)=\frac{b_{0} z^{m}+b_{1} z^{m-1}+\cdots+b_{m}}{z^{n}+a_{1} z^{n-1}+a_{2} z^{n-2}+\cdots+a_{n}}
$$

is the transfer function of the model for the parameter vector $(\mathbf{a}, \mathbf{b})=\left(a_{1}, \ldots, a_{n}, b_{0}, \ldots, b_{m}\right)$, with $n>m$.

Suppose that the time delay of the system is given by $d=$ $n-m-1$; thus (1) is equivalent to

$$
\begin{aligned}
\Delta y(k)= & b_{0} \Delta u(k-(d+1))+b_{1} \Delta u(k-(d+2))+\cdots \\
& +b_{m} \Delta u(k-n)-a_{1} \Delta y(k-1)-\cdots \\
& -a_{n} \Delta y(k-n)
\end{aligned}
$$

A large diversity of real processes can be modeled using CARIMA models, for instance, unmanned seaplanes [29], stirred tank reactors [30], vehicle yaw [31], gas engines [32], and distillation columns $[33,34]$. Therefore, the development of control design techniques that are suitable for those models is relevant.

It is assumed that the process to be controlled is described by the model (1), subject to uncertainties on the coefficients of the transfer function, with each parameter $a_{j}$ and $b_{i}$ belonging to its respective uncertainty interval $\underline{a}_{j} \leq a_{j} \leq \bar{a}_{j}$ and $\underline{b}_{i} \leq$ $b_{i} \leq \bar{b}_{i}$. The box set $\mathscr{C}$ given by

$$
\begin{aligned}
\mathscr{C} & =\left\{(\mathbf{a}, \mathbf{b})=\left(a_{1}, \ldots, a_{n}, b_{0}, \ldots, b_{m}\right) \mid \underline{a}_{j} \leq a_{j} \leq \bar{a}_{j}, \underline{b}_{i}\right. \\
& \left.\leq b_{i} \leq \bar{b}_{i}\right\}
\end{aligned}
$$

describes the possible instances of the system parameter vector. It is assumed here that $\underline{a}_{j} \bar{a}_{j} \geq 0 \forall j=1, \ldots, n$ and $\underline{b}_{i} \bar{b}_{i} \geq 0 \forall i=1, \ldots, m$.

System (1) with uncertain parameters described by (4) will be controlled by a Model Predictive Control (MPC) scheme, for which the following notation is employed: $w(k)$ is the reference which $y(k)$ should track; $\Delta y(k+i \mid k)$ is the predicted value of the output increment $\Delta y$ on time $k+i$, calculated on time $k$, for $i=\left\{1, \ldots, H_{p}\right\}$, where $H_{p}$ is the prediction horizon; $\Delta u(k+i \mid k)$ is the future control signal increment at time $k+i$, used in the output increment predictions, for $i=\left\{0, \ldots, H_{c}-1\right\}$, where $H_{c}$ is the control horizon $\left(H_{c} \leq H_{p}\right)$.

Different cost functions can be adopted for the definition of an MPC strategy which works along with the stability constraints that are proposed in this paper. For simplicity, the cost function $J$ of the MPC is assumed to be the traditional quadratic function that considers the error between the 
prediction $\widehat{y}(k+j \mid k)$ and a known reference $w(k+j)$ and the control input increment $\Delta u(k)$ :

$$
\begin{aligned}
J= & \sum_{j=1}^{H_{p}} \delta(j)(\hat{y}(k+j \mid k)-w(k+j))^{2} \\
& +\sum_{i=0}^{H_{c}-1} \lambda(i)(\Delta u(k+i \mid k))^{2}
\end{aligned}
$$

where $\delta(j)$ and $\lambda(i)$ are the weighting sequences of the error and the control effort, respectively. The following min-max problem is considered in the MPC, for the plant model with parameter uncertainty:

$$
\min _{\Delta u} \max _{(\mathbf{a}, \mathbf{b}) \in \mathscr{C}} J(\Delta u, \mathbf{a}, \mathbf{b}) .
$$

An MPC employing the min-max objective function (6) was proposed in [13]. That control, in its unconstrained version, is named here as the RMPC (MPC with robust min-max approach). The MPC to be considered in this paper will be based on the RMPC, because the min-max index in (6) is suitable for dealing with set-bounded uncertainties. However it should be noticed that other indices could be defined, for instance, based on the expectation of the values of the uncertain parameters. Those alternative indices are expected to lead to better performances, since they are not assigned to the role of ensuring the system robustness in the context of the proposed methodology, which will enforce stability using constraints. The definition of those alternative indices is left as a theme for future research.

The problem to be solved in this paper is defined as follows [35].

Definition 1 (asymptotic convergence problem). Consider system (3) with uncertain parameters as described by (4), with any given initial condition $\{\Delta u(-m), \ldots, \Delta u(0)$, $\Delta y(-n), \ldots, \Delta y(0)\}$, and a given constant reference signal $w(k)=\rho$, with $\rho \in \mathbb{R}$. Find a sequence of control input increments $\Delta u(k \mid 0)$, for $k>0$, such that

$$
\lim _{k \rightarrow \infty} y(k \mid 0)-w(k)=0
$$

for all $(\mathbf{a}, \mathbf{b}) \in \mathscr{C}$.

For the sake of simplicity, the reference input $w(k)$ is assumed to be zero in the development that follows. More general reference signals can be tackled according to the guidelines presented, for instance, in [21].

\section{Convergence Constraints}

The results to be established in the sequel rely on the following lemma.

Lemma 2. Suppose the output sequence $y(k)$ of a discrete-time system satisfies the following condition:

$$
|y(k+r)|<\varepsilon|y(k)|, \text { with } 0<\varepsilon<1 \forall k,
$$

where $r$ is a positive integer. Then, $|y(k)| \rightarrow 0$ when $k \rightarrow \infty$.
Proof. This comes directly from $\lim _{k \rightarrow \infty} \varepsilon^{k}=0 \forall k \in[0,1)$.

This lemma states a standard sufficient condition for the convergence of a sequence. The basic idea here is to impose condition (8) to the plant output, propagating it as a constraint for the values of the input increments $\Delta u(k)$ such that the predicted output satisfies (8). The plant output should stay as close as possible to a reference signal $w(k)$. The following convergence condition is imposed:

$$
|y(k+r \mid k)-w(k+r)|<\epsilon|y(k)-w(k)|,
$$

where $0<\epsilon<1$ and $r$ is a positive integer, which leads to $|y(k)-w(k)| \rightarrow 0$. Considering $k$ the current time instant, a sequence of future input increments $\Delta \mathbf{u}=\{\Delta u(k \mid k), \Delta u(k+$ $\left.1 \mid k), \ldots, \Delta u\left(k+H_{c}-1 \mid k\right)\right\}$ should be determined such that (9) is satisfied. The computation of such a sequence will be performed using a state-space description of the system. Let the following matrices be defined:

$\mathbf{0}_{(i, j)}$ : matrix with $i$ rows and $j$ columns, with all entries equal to 0 .

$\mathbf{I}_{j}$ : identity matrix with $j$ rows and columns.

$$
\begin{aligned}
& \mathbf{S}_{j}=\left[\begin{array}{ll}
\mathbf{0}_{(j, 1)} & \mathbf{I}_{j}
\end{array}\right] . \\
& \mathbf{a}=\left[\begin{array}{lllll}
a_{1} & a_{2} & \cdots & a_{n-1} & a_{n}
\end{array}\right] .
\end{aligned}
$$

Define also the vectors:

$$
\mathbf{z}(k)=\left[\begin{array}{c}
\Delta y(k) \\
\Delta y(k-1) \\
\vdots \\
\Delta y(k-(n-1)) \\
\Delta u(k-1) \\
\Delta u(k-2) \\
\vdots \\
\Delta u(k-(n-1))
\end{array}\right]_{(m+n+d) \times 1},
$$

In addition, the matrices $\Phi$ and $\Omega$ are defined in different ways, depending on the value of the time delay $d$. For $d>0$,

$$
\begin{aligned}
& \mathbf{b}=\left[\begin{array}{lllll}
b_{0} & b_{1} & \cdots & b_{m-1} & b_{m}
\end{array}\right], \\
& \mathbf{b}^{\prime}=\left[\begin{array}{ll}
\mathbf{0}_{(1, d-1)} & \mathbf{b}
\end{array}\right], \\
& \Phi=\left[\begin{array}{cc}
-\mathbf{a} & \mathbf{b}^{\prime} \\
\mathbf{S}_{n-1} & \mathbf{0}_{(n-1, m+d)} \\
\mathbf{0}_{(1, n-1)} & \mathbf{0}_{(1, m+d)} \\
\mathbf{0}_{(m+d-1, n)} & \mathbf{S}_{m+d-1}
\end{array}\right] \text {, } \\
& \Omega=\left[\begin{array}{lll}
\mathbf{0}_{(n, 1)} & 1 & \mathbf{0}_{(m+d-1,1)}
\end{array}\right]^{T} .
\end{aligned}
$$


For $d=0$,

$$
\begin{aligned}
& \mathbf{b}=\left[\begin{array}{llll}
b_{1} & \cdots & b_{m-1} & b_{m}
\end{array}\right], \\
& \Phi=\left[\begin{array}{cc}
-\mathbf{a} & \mathbf{b} \\
\mathbf{S}_{n-1} & \mathbf{0}_{(n-1, m)} \\
\mathbf{0}_{(1, n)} & \mathbf{0}_{(1, m)} \\
\mathbf{0}_{(m-1, n)} & \mathbf{S}_{m-1}
\end{array}\right], \\
& \Omega=\left[\begin{array}{llll}
b_{0} & \mathbf{0}_{(n-1,1)} & 1 & \mathbf{0}_{(m-1,1)}
\end{array}\right]^{T} \text {. }
\end{aligned}
$$

The system represented by the transfer function (2) is equivalent to the space-state system given by

$$
\begin{aligned}
\mathbf{z}(k+1) & =\Phi \mathbf{z}(k)+\Omega \Delta u(k), \\
\Delta y(k+1) & =\Psi \mathbf{z}(k+1) .
\end{aligned}
$$

Now define the matrix

$$
\Theta_{r}=\left[\begin{array}{llllll}
\Phi^{r-1} \Omega & \Phi^{r-2} \Omega & \cdots & \Phi \Omega & \Omega
\end{array}\right]
$$

and the decision variable vector

$$
\begin{aligned}
& \Delta \mathbf{u}_{r}(k) \\
& =\left[\begin{array}{lllll}
\Delta u(k \mid k) & \Delta u(k+1 \mid k) & \cdots & \Delta u(k+r-1 \mid k)
\end{array}\right]^{T} .
\end{aligned}
$$

The following lemma can be stated as a result of the recursive application of (13).

Lemma 3. The r-step-ahead prediction model of system (2) is given by

$$
\begin{aligned}
\mathbf{z}(k+r) & =\Phi^{r} \mathbf{z}(k)+\Theta_{r} \Delta \mathbf{u}_{r}(k), \\
\Delta y(k+r) & =\Psi \mathbf{z}(k+r) .
\end{aligned}
$$

Proof. Expression (16) comes as a direct composition of (13) applied on $r$ consecutive steps, with the replacement of expressions (14) and (15) in the suitable places.

The matrices $\Phi^{r}$ and $\Theta_{r}$ depend on the uncertain parameters $a_{j}$ and $b_{i}$. On the instant $k$, the vector $\mathbf{z}(k)$ is composed of known scalars (past values of $u(\cdot)$ and $y(\cdot)$, up to instant $k$ ). The variable $y(k+r)$ depends on $\Phi^{r}, \Theta_{r}$ and on the decision variable vector $\Delta \mathbf{u}_{r}(k)$, which should be specified such that (9) holds. The following lemma, stated under the assumption that there is no system uncertainty, constitutes a well-known result related to deadbeat controllers which is presented here as a bound for the achievable system performance.

Lemma 4. Assume that system (13) is controllable. In this case, for any initial condition $\mathbf{z}(k)$, there exists a decision variable vector $\Delta \mathbf{u}_{r}(k)$ with $r \leq n+m$ such that $\mathbf{z}(k+r)=0$.

Proof. It should be noticed that $\Theta_{r}$ becomes equivalent to the controllability matrix of the system when $r=n+m$. In the case of a controllable system, the controllability matrix is a full-rank square matrix of size $(n+m) \times(n+m)$. Therefore, when $r=n+m$ it will be possible to state $\{u(k), u(k+$ $1), \ldots, u(k+r-1)\}$ such that

$$
\Delta \mathbf{u}_{(n+m)}(k)=-\Theta_{(n+m)}^{-1} \Phi^{(n+m)} \mathbf{z}(k) .
$$

In some special cases, it might be possible that a number of steps smaller than $n+m$ become enough, which completes the proof.

Considering the uncertain parameter case, a control strategy that would be analogous to the deadbeat control may be represented by

$$
\Delta \mathbf{u}_{r}^{*}=\arg \left(\min _{\Delta \mathbf{u}_{r}} \max _{\mathscr{C}}|y(k+r \mid k)|\right) .
$$

Lemma 4 suggests that the choice $r=m+n$ would be reasonable, even for the case of systems with uncertainty, because with a smaller value for $r$ the control action might be unable to produce an arbitrary contraction of the output error. A more relaxed design formulation may be stated, requiring only the feasibility of condition (9):

$$
\Delta \mathbf{u}_{r}(k):\left(\max _{\mathscr{C}}|y(k+r \mid k)|\right)<\epsilon|y(k)| .
$$

Expression (19) reduces to (18) when $\epsilon$ reaches its minimum value that still results in a feasible problem. In order to solve (19), it is worthy to note that

$$
\begin{aligned}
y(k+r \mid k)= & \Delta y(k+r \mid k)+\Delta y(k+r-1 \mid k) \\
& +\cdots+\Delta y(k+1 \mid k)+y(k) .
\end{aligned}
$$

Using (16), (20) can be rewritten as

$$
\begin{aligned}
y(k+r \mid k)= & \Psi\left(\Phi^{r}+\Phi^{r-1}+\cdots+\Phi\right) \mathbf{z}(k) \\
& +\Psi \widehat{\boldsymbol{\Theta}} \Delta \mathbf{u}_{r}(k)+y(k)
\end{aligned}
$$

where $\widehat{\Theta}$ is the matrix:

$$
\widehat{\boldsymbol{\Theta}}=\left[\left(\Phi^{r-1}+\Phi^{r-2}+\cdots+\Phi+\mathbf{I}_{m+n+d}\right) \Omega\left(\Phi^{r-2}+\Phi^{r-3}+\cdots+\Phi+\mathbf{I}_{m+n+d}\right) \Omega \cdots\left(\Phi+\mathbf{I}_{m+n+d}\right) \Omega \Omega\right]
$$


Consider

$$
\begin{aligned}
& \Lambda=\Psi\left(\Phi^{r}+\Phi^{r-1}+\cdots+\Phi\right) \mathbf{z}(k)+y(k), \\
& \Gamma_{r}=\Psi \widehat{\boldsymbol{\Theta}}=\left[\begin{array}{lllll}
\gamma_{r} & \gamma_{r-1} & \cdots & \gamma_{2} & \gamma_{1}
\end{array}\right] .
\end{aligned}
$$

With the use of optimization tools, it is possible to obtain

$$
\begin{aligned}
& \underline{\Lambda}=\min _{\mathscr{C}} \Psi\left(\Phi^{r}+\Phi^{r-1}+\cdots+\Phi\right) \mathbf{z}(k)+y(k), \\
& \bar{\Lambda}=\max _{\mathscr{C}} \Psi\left(\Phi^{r}+\Phi^{r-1}+\cdots+\Phi\right) \mathbf{z}(k)+y(k) .
\end{aligned}
$$

Suppose, without loss of generality, that the reference signal $w(k)$ is zero. So, the stability condition (9) can be rewritten as

$$
\left|\Gamma_{r} \Delta \mathbf{u}_{r}(k)+\Lambda\right|<\epsilon|y(k)|
$$

Equivalently,

$$
-\epsilon|y(k)|<\Gamma_{r} \Delta \mathbf{u}_{r}(k)+\Lambda<\epsilon|y(k)|,
$$

which leads to

$$
-\epsilon|y(k)|-\underline{\Lambda} \leq \Gamma_{r} \Delta \mathbf{u}_{r}(k) \leq \epsilon|y(k)|-\bar{\Lambda}
$$

The coefficients $\gamma_{i}$ of the vector $\Gamma_{r}$ are limited by

$$
\begin{aligned}
\overline{\gamma_{i}} & =\max _{\mathscr{C}} \gamma_{i} \\
\underline{\gamma_{i}} & =\min _{\mathscr{C}} \gamma_{i} \\
& \quad i=1, \ldots, r .
\end{aligned}
$$

Consider the matrix $\mathbf{M}$, whose rows correspond to all possible combinations of minimum and maximum values of the components of vector $\Gamma_{r}$ :

$$
\mathbf{M}=\left[\begin{array}{ccccc}
\overline{\gamma_{r}} & \overline{\gamma_{r-1}} & \cdots & \overline{\gamma_{2}} & \overline{\gamma_{1}} \\
\overline{\gamma_{r}} & \overline{\gamma_{r-1}} & \cdots & \overline{\gamma_{2}} & \underline{\gamma_{1}} \\
\overline{\gamma_{r}} & \overline{\gamma_{r-1}} & \cdots & \underline{\gamma_{2}} & \overline{\gamma_{1}} \\
\overline{\gamma_{r}} & \overline{\gamma_{r-1}} & \cdots & \underline{\gamma_{2}} & \underline{\gamma_{1}} \\
\vdots & \vdots & \cdots & \vdots & \vdots \\
\underline{\gamma_{r}} & \underline{\gamma_{r-1}} & \cdots & \overline{\gamma_{2}} & \underline{\gamma_{1}} \\
\underline{\gamma_{r}} & \underline{\gamma_{r-1}} & \cdots & \underline{\gamma_{2}} & \overline{\gamma_{1}} \\
\underline{\gamma_{r}} & \underline{\gamma_{r-1}} & \cdots & \underline{\gamma_{2}} & \underline{\gamma_{1}}
\end{array}\right] .
$$

Note that

$$
\begin{array}{ll}
\mathbf{M} \Delta \mathbf{u}_{r}(k) \leq \mathbf{1}_{\left(2^{r}, 1\right)}(\epsilon|y(k)|-\bar{\Lambda}) \Longrightarrow & \\
\Gamma_{r} \Delta \mathbf{u}_{r}(k) \leq \epsilon|y(k)|-\bar{\Lambda}, & \forall(\mathbf{a}, \mathbf{b}) \in \mathscr{C}, \\
\mathbf{M} \Delta \mathbf{u}_{r}(k) \geq \mathbf{1}_{\left(2^{r}, 1\right)}(-\epsilon|y(k)|-\underline{\Lambda}) \Longrightarrow & \\
\Gamma_{r} \Delta \mathbf{u}_{r}(k) \geq-\epsilon|y(k)|-\underline{\Lambda}, & \\
& \forall(\mathbf{a}, \mathbf{b}) \in \mathscr{C} .
\end{array}
$$

On this point, it is possible to state Theorem 5, which constitutes the main result of this paper.
Theorem 5. Let $\Delta \mathbf{u}_{r}(k)$ denote the vector of the next $r$ control input increments to be applied to system (2) after instant $k$. If

$$
\left[\begin{array}{c}
\mathbf{M} \\
-\mathbf{M}
\end{array}\right] \Delta \mathbf{u}_{r}(k) \leq\left[\begin{array}{l}
\mathbf{1}_{\left(2^{r}, 1\right)}(\epsilon|y(k)|-\bar{\Lambda}) \\
\mathbf{1}_{\left(2^{r}, 1\right)}(\epsilon|y(k)|+\underline{\Lambda})
\end{array}\right]
$$

holds, then the condition

$$
|y(k+r \mid k)|<\epsilon|y(k)|
$$

is satisfied, for all $(\mathbf{a}, \mathbf{b}) \in \mathscr{C}$.

Proof. The proof of this theorem is stated as the sequence of expressions, from expression (25) to expression (31).

The RMPC, with objective function stated in (6), jointly with the stability constraints (32), will be called the RMPC-SC (RMPC with Stability Constraints). The RMPC-SC algorithm is presented in Algorithm 1.

In practice, the feasibility of inequality (32) will depend on the size of the model uncertainty. For systems with small uncertainty sets, those conditions will be satisfied easily, while systems with large uncertainties may be unable to achieve the feasibility of that inequality. This last case corresponds to the situation in which there is no guarantee that a control input sequence will be able to enforce the decrease of the amplitude of the output signal for all instances of such systems.

An important issue in MPC algorithms is the recursive feasibility, which means the guarantee that the system state resulting from an iteration of the MPC will always be feasible for the problem constraints, provided that the previous iteration was also performed from a feasible initial state.

Definition 6 (recursive feasibility). Let $\Delta \mathbf{u}_{r}(k)$ be defined according to (15). Then, if the relation

$$
\begin{aligned}
& \left\{\Delta \mathbf{u}_{r}(k) \text { satisfies }(32)\right. \\
& \left.\quad \Longrightarrow \Delta \mathbf{u}_{r}(k+1) \text { satisfies }(32)\right\}
\end{aligned}
$$

holds, Algorithm 1 is said to be recursively feasible.

The following lemma states some sufficient conditions for recursive feasibility of RMPC-SC.

Lemma 7. Consider a system described by (1) and (2), with parameters belonging to the set $\mathscr{C}$, described by (4), being controlled by Algorithm 1. Let $\rho>0$ be a scalar such that $\rho \geq \bar{a}_{j}-\underline{a}_{j} \forall j=1, \ldots, n$ and $\rho \geq \bar{b}_{i}-\underline{b}_{i} \forall i=1, \ldots, m$. Then, consider the following:

(i) If the set $\mathscr{C}$ represents a precisely known model, that is, $\underline{a}_{j}=\bar{a}_{j} \forall j=1, \ldots, n$ and $\underline{b}_{i}=\bar{b}_{i} \forall i=1, \ldots, m$, then Algorithm 1 is guaranteed to have recursive feasibility for some $r \leq n+m$ and for any $\epsilon \in(0,1)$.

(ii) For all $\epsilon \in(0,1)$ there exists a $\rho$ which makes Algorithm 1 recursively feasible.

Proof. Statement (i) comes from the fact that, in the case of a precisely known system, the deadbeat control (Lemma 4) 
(1) Initialization: Define the model and the uncertainty set $\mathscr{C}$, also the control parameters $H_{p}, H_{c}, \epsilon$ and $r$, the reference signal $w$ and the initial conditions $\mathbf{z}$.

(2) $k \leftarrow 0$

(3) Determine the matrix $\mathbf{M}$ according to (29).

(4) while $k<k_{\max }$ do

(5) Calculate $\bar{\Lambda}$ and $\underline{\Lambda}$, according to (24).

(6) Calculate $\Delta \mathbf{u}_{r}(k)$ optimizing (6), subject to constraints (32) and possibly other constraints related to the specific problem.

(7) $u(k) \leftarrow \Delta u(k)+u(k-1)$

(8) Apply $u(k)$ to the plant input, and get $y(k)$ from the plant output.

(9) $k \leftarrow k+1$

(10) end while

Algorithm 1: RMPC control with Stability Constraints (RMPC-SC).

can be applied. Therefore, the output can be brought from any initial state to zero in up to $n+m$ steps, so (32) will be feasible for any $\epsilon \in(0,1)$. Statement (ii) is established by a simple continuity argument which is based on the fact that the mapping from $(\mathbf{a}, \mathbf{b})$ to $\mathbf{z}(k)$ defined implicitly in (16) is continuous. This means that as $(\mathbf{a}, \mathbf{b})$ depart from their nominal values and their uncertainty intervals grow, the value of $\epsilon$ which is necessary for making (32) feasible will be greater. Clearly, for a sufficiently large $\rho$, there will be no $\epsilon<1$ that makes (32) feasible.

Some additional comments are necessary. First, there is no guarantee that the stability constraints are compatible with other constraints that may become necessary in a particular situation. If more constraints are necessary, the recursive feasibility of the resulting MPC should be studied for that particular case.

Also, as can be inferred from the proof of Lemma 7, in the case of uncertain systems whose parameters have arbitrary uncertainty intervals, the recursive feasibility of the constraints (32) may not hold. When the uncertainty intervals are large it becomes possible that different system instances belonging to the uncertainty set are conflicting, requiring quite different control inputs to produce the effect of decreasing the absolute value of the system output. Rigorously, this situation means that it would be necessary to reduce the uncertainty intervals, in order to allow the stabilization condition to be verified in all time instants. This may be performed either by going back to the system identification phase, in order to find more precise models for the plant, or by an online refinement of the model that discards some regions of the uncertainty set that are inconsistent with the observed plant behavior. The issue of assessing model consistency with data has been studied in some detail in Barbosa et al. [36].

\section{Simulation Results}

In this section, the performance of the RMPC-SC technique is first evaluated under some different circumstances. Afterwards, the RMPC-SC is compared with four other techniques of predictive control: the basic MPC and the GPC [37], both nonrobust, the RMPC (an MPC with robust min-max approach) [13], and the RGPC (robust GPC) proposed in $[19,20]$.

In the simulation studies presented here, the value for the parameter $\delta(j)$ of the objective function (5) is kept constant as $\delta(j)=1$ for all methods. The aim of the design task considered here is to find the control sequence $\Delta u=\Delta u(k+j \mid$ $k), j=0, \ldots, H_{c}-1$. In the basic MPC and GPC and in the RGPC, a nominal model is considered for the prediction.

The RGPC represents an interesting benchmark for comparison with RMPC-SC, since it is conceived with the purpose of providing robustness against norm-bounded disturbances. The diagram of RGPC is presented in Figure 1. In the diagram, the low-pass filter provides the additional degrees of freedom that are used in order to achieve the robustness in the case of an uncertain model.

Two examples are considered in the simulation studies. The first one is the classic angular positioning system stated by Kwakernaak and Sivan [38]. The second example refers to a simulated plant model, which was based on the problem posed by Ramírez and Camacho [13], with constraints in the increment input signal. The simulations were performed in Matlab (R2013a). It was considered the initial condition $u(0)=0.05$ and a reference signal $w$ constant and null.

4.1. Problem 1. The system consists of a rotating antenna at the origin of the plane, driven by an electric motor. The control problem is to provide an input voltage $(u V)$ to the motor, so as to direct the antenna to an object that moves on the plane [39]. The antenna rotation can be described by the following model:

$$
\theta(k)=\frac{0.0078}{z^{2}+(0.1 \alpha(k)-2) z+(1-0.1 \alpha(k))} u(k),
$$

where the sampling period is $0.1 \mathrm{~s}, \theta(k)$ is the angular position $(\mathrm{rad})$, and $0.1 \mathrm{~s}^{-1} \leq \alpha(k) \leq 10 \mathrm{~s}^{-1}$. The parameter $\alpha(k)$ is proportional to the coefficient of viscous friction in the rotating parts of the antenna and is assumed to be arbitrarily time-varying in the interval $[0.1,10]$. In the model used in MPC and GPC, $\alpha(k)$ is fixed in the nominal value $\alpha_{n}(k)$.

4.1.1. RMPC-SC Performance. Simulations were first performed for various values of $\alpha(k)$, equally spaced within 


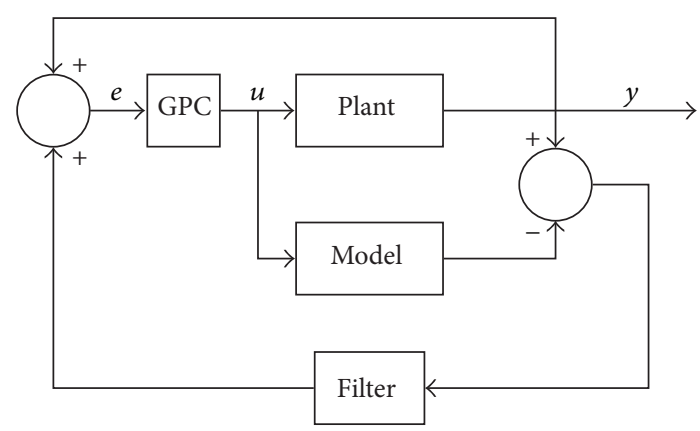

FIGURE 1: Diagram of RGPC.

the range $[0.1,10]$. The RMPC-SC was applied using the prediction and control horizons, $H_{p}=12$ and $H_{c}=6$, respectively, and $r=3$. The values of the control parameters $\epsilon$ and $\lambda$ have been taken within the ranges $[0.7 ; 0.9]$ and $[1,3]$, respectively. Figure 2 shows the results obtained, with the stabilization of the plant in all cases.

The RMPC-SC controller was also simulated for the system represented by the model (35) with $\alpha(k)$ varying randomly with time, within the uncertainty interval. Figure 3 shows an instance of the response of the LTV system controlled by RMPC-SC, with control parameters $H_{p}=12, H_{c}=$ $6, r=3, \epsilon=0.8$, and $\lambda=3$. In fifty other simulations with other realizations of the uncertain time-varying parameter the system converged.

In order to analyze how the variation of the parameter $\epsilon$ interferes in the response of the system, the RMPC-SC was applied to control the plant (35), with $\alpha(k)=10$, using different values for $\epsilon$. For each value of $\epsilon$ in the set $\{0.4 ; 0.6 ; 0.8 ; 0.9\}$, the RMPC-SC was applied with $H_{p}=12$, $H_{c}=6, \lambda=3$, and $r=3$, and the simulation results are shown in Figure 4. A better performance is observed, in this case, when $\epsilon=0.9$.

4.1.2. Comparison Studies. Figure 5 presents the results obtained by RMPC-SC, RGPC, MPC, and GPC controllers for the case $\alpha(k)=0.1$. The GPC, MPC, and RMPC considered $\alpha_{n}(k)=1$. As can be seen in Figure 5, the methods RMPC-SC, MPC, GPC, and RGPC achieved convergence, with the best performance presented by MPC. The RMPC became unstable in this case.

As can be seen in Figure 5, all methods except RMPC showed a good control performance. The good results presented by GPC, RGPC, and MPC may be explained by the proximity of the plant model to the nominal model. By taking $\alpha_{n}(k)=10$, the system controlled by GPC, RGPC, and MPC becomes unstable.

The values of the control parameters used by each method to obtain the results depicted in Figure 5 are described in Table 1.

For $\alpha(k)=10$, all methods stabilize the plant, with a faster response obtained by the system controlled by RMPC-SC, as shown in Figure 6. It used $\alpha_{n}(k)=0.1$ in GPC, RGPC, and MPC. The values of the control parameters used in this case are described in Table 2.

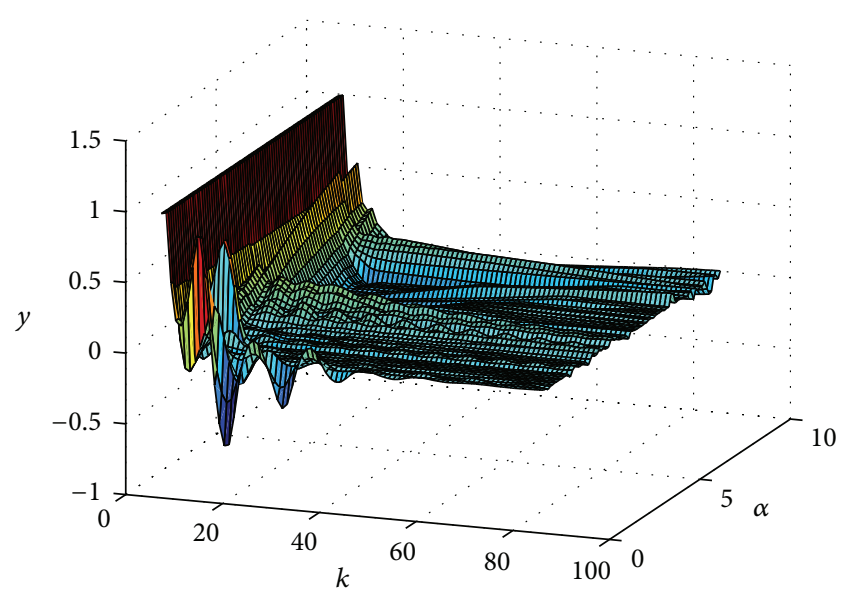

FIGURE 2: RMPC-SC with $\alpha(k) \in[0.1 ; 10]$.

TABLE 1: Control parameters of RMPC-SC, RMPC, RGPC, GPC, and MPC for the simulated plant with $\alpha(k)=0.1$. In this table, $\mathcal{O}$ means the filter order and $w_{c}$ means the filter cutoff frequency, for RGPC.

\begin{tabular}{lccccccc}
\hline & $H_{p}$ & $H_{c}$ & $\lambda$ & $\epsilon$ & $r$ & $\mathcal{O}$ & $w_{c}$ \\
\hline RMPC-SC & 12 & 6 & 1 & 0.6 & 3 & - & - \\
RMPC & 5 & 3 & 3 & - & - & - & - \\
RGPC & 14 & 10 & 1 & - & - & 2 & 0.85 \\
GPC & 14 & 10 & - & - & - & - & - \\
MPC & 12 & 6 & 0.05 & - & - & - & - \\
\hline
\end{tabular}

4.2. Problem 2. The following transfer function plant model with two uncertain coefficients was based on [13]:

$$
T(z)=\frac{b z+1}{z^{2}-1.9 z+a},
$$

with the coefficients $a$ and $b$ in the box:

$$
\mathscr{C}=\left\{\begin{array}{l}
0.7 \leq a \leq 1 \\
0.5 \leq b \leq 1.5 .
\end{array}\right.
$$

The plant is also subject to the constraint $-2 \leq \Delta u \leq 2$.

The following control parameters were used by RMPC, RGPC, and RMPC-SC: $H_{p}=8, H_{c}=4$, and $\lambda=1$. The RMPC-SC also used $\epsilon=0.9$ and $r=3$. The RGPC used $\mathcal{O}=2$ and $w_{c}=0.9$. Due to the bad results obtained by the MPC and GPC controllers, the performances of those methods are not presented here.

The following nonminimum phase instance of the plant model was considered first:

$$
T_{p 1}(z)=\frac{0.5 z+1}{z^{2}-1.9 z+1} .
$$

The RMPC and RMPC-SC methods were applied in the control of the plant (38) and have shown satisfactory results, as can be seen in Figure 7. The RGPC was applied on the same plant and has shown less satisfactory results, with a highfrequency control signal, as can be seen in Figure 8. In this case, RGPC used $\lambda=5$ and considered the model described in (36) as a nominal model, with $a=1$ and $b=1$. 


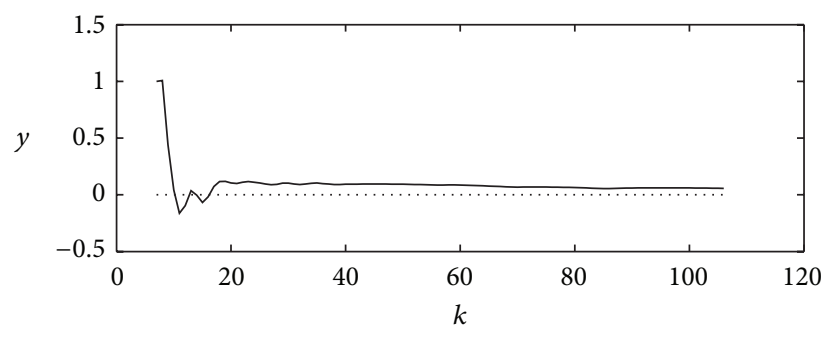

— RMPC-SC

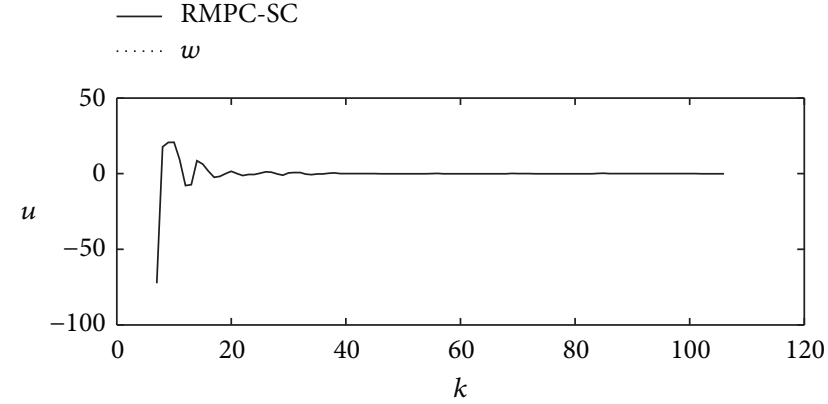

- RMPC-SC

FIGURE 3: RMPC-SC with LTV model.
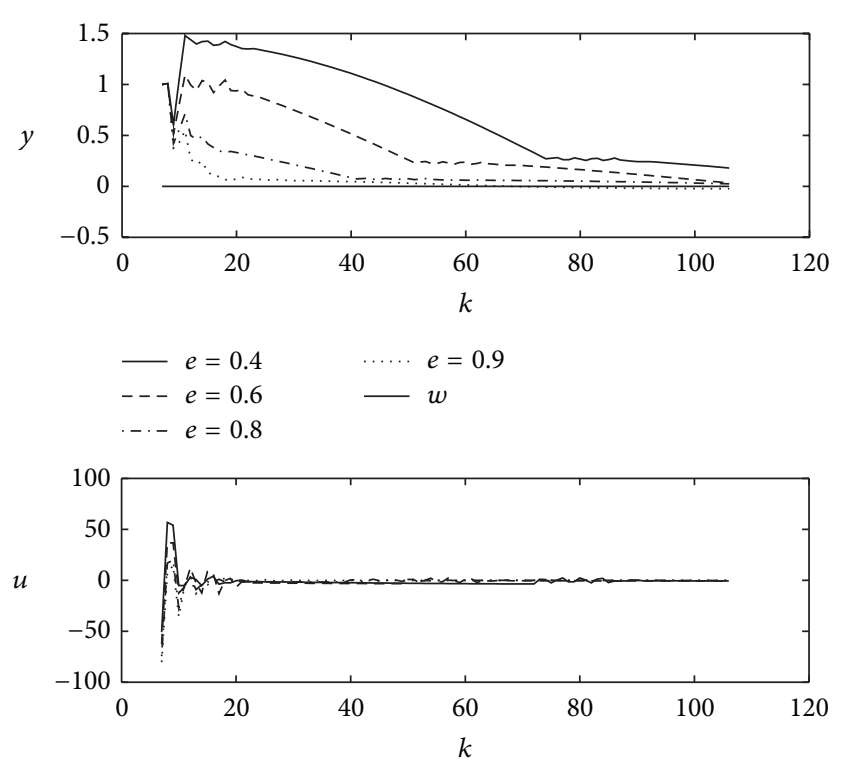

$-e=0.4 \quad--e=0.8$

$--e=0.6 \quad$ …e $e=0.9$

FIGURE 4: System response of RMPC-SC for different values of $\epsilon$, with $\alpha(k)=10$.

The following unstable model instance of the plant was also considered:

$$
T_{p 2}(z)=\frac{1.5 z+1}{z^{2}-1.9 z+0.7}
$$

The RMPC-SC showed a satisfactory result in this case, as can be seen in Figure 9, while RMPC and RGPC did not achieve stability.
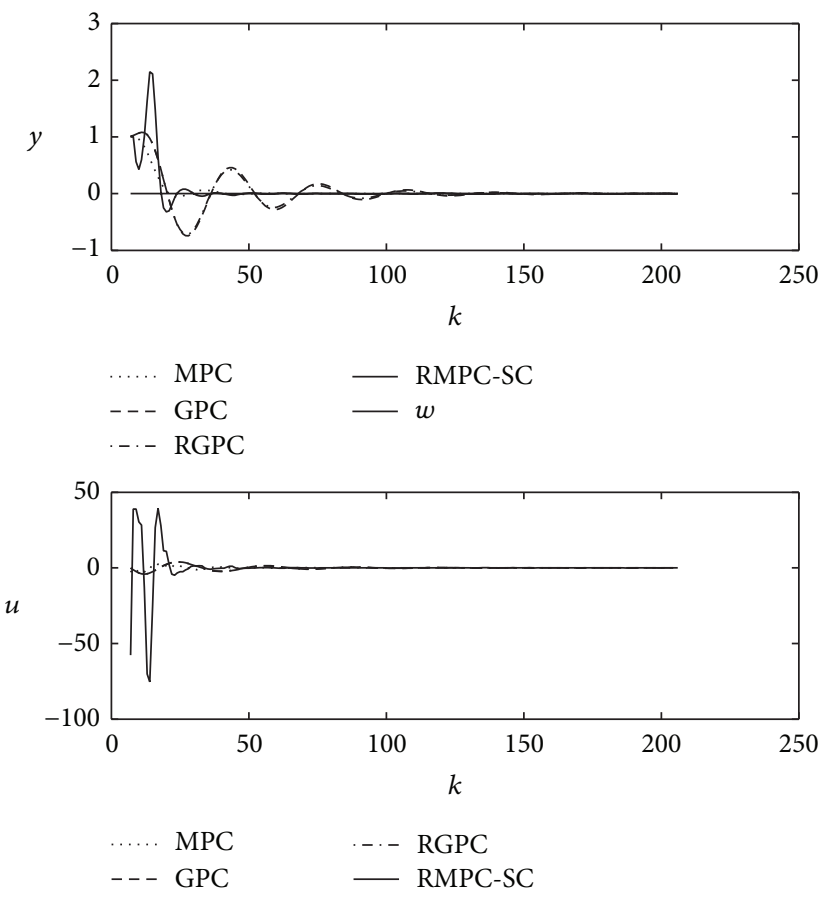

FIGURE 5: MPC $\times$ GPC $\times$ RGPC $\times$ RMPC-SC with $\alpha(k)=0.1$ and $\alpha_{n}(k)=1$.

TABLE 2: Control parameters of RMPC-SC, RMPC, RGPC, GPC, and MPC for the simulated plant with $\alpha(k)=10$ and $\alpha_{n}(k)=0.1$. In this table, $\mathcal{O}$ means the filter order and $w_{c}$ means the filter cutoff frequency, for RGPC.

\begin{tabular}{lccccccc}
\hline & $H_{p}$ & $H_{c}$ & $\lambda$ & $\epsilon$ & $r$ & $\mathcal{O}$ & $w_{c}$ \\
\hline RMPC-SC & 12 & 6 & 3 & 0.9 & 3 & - & - \\
RMPC & 6 & 4 & 1 & - & - & - & - \\
RGPC & 12 & 6 & 10 & - & - & 2 & 0.9 \\
GPC & 12 & 6 & - & - & - & - & - \\
MPC & 12 & 6 & 0.05 & - & - & - & - \\
\hline
\end{tabular}

The RMPC-SC and RMPC controllers were applied to the plant (36) for different sets of values of the coefficients $a$ and $b$ in $\mathscr{C}: 36$ pairs of coefficients $(a, b)$ were generated, with $a \in\{0.5 ; 0.7 ; 0.9 ; 1.1 ; 1.3 ; 1.5\}$ and $b \in$ $\{0.7 ; 0.76 ; 0.82 ; 0.88 ; 0.94 ; 1\}$. A controller was considered to be stabilizing when the mean of difference $(y(k)-w(k))$ was lower than 0.01 for $N / 2 \leq k \leq N$, where $N$ is the number of steps of the algorithm (in this example it used $N=$ 100). Figure 10 shows the instances for which each method achieved satisfactory results. It can be seen that the RMPC-SC achieves stabilization for $35 / 36$ of the system instances, while RMPC stabilized the system in only $26 / 36$ of the instances. There was only one instance of the pair $(a, b)$ for which the RMPC-SC did not get a good performance: the pair $(a, b)=$ $(0.7,0.5)$.

The same analysis was performed in order to compare the stabilization provided by RMPC-SC and RGPC controllers, for the same plant (36), considering the same grid of parameter values. In this analysis, the parameters of RGPC were 

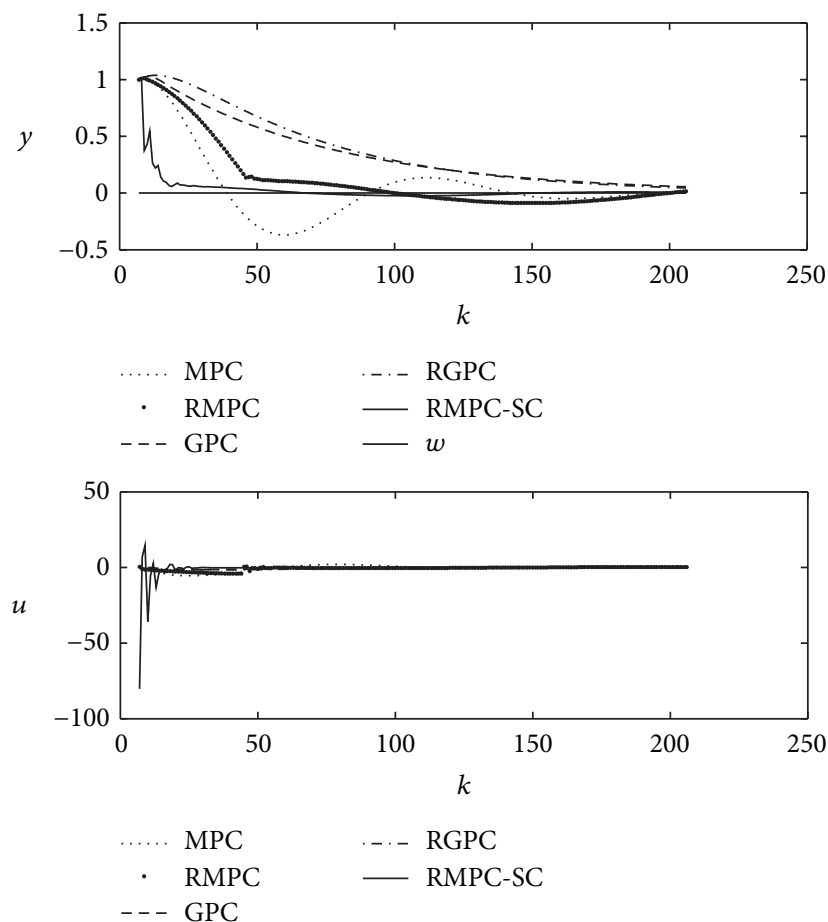

FiguRE 6: MPC $\times$ RMPC $\times$ GPC $\times$ RGPC $\times$ RMPC-SC: $\alpha(k)=10$ and $\alpha_{n}(k)=0.1$.

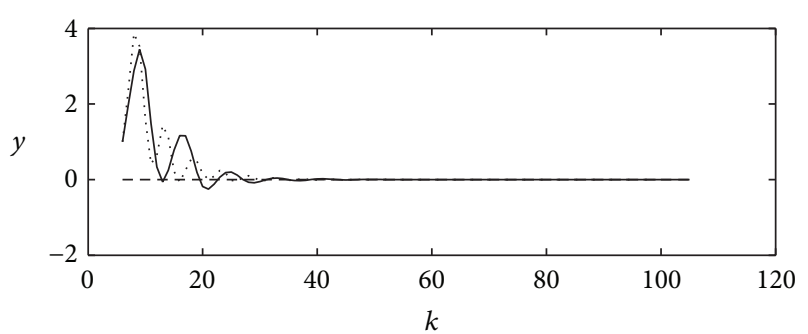

- RMPC-SC

RMPC

$---w$

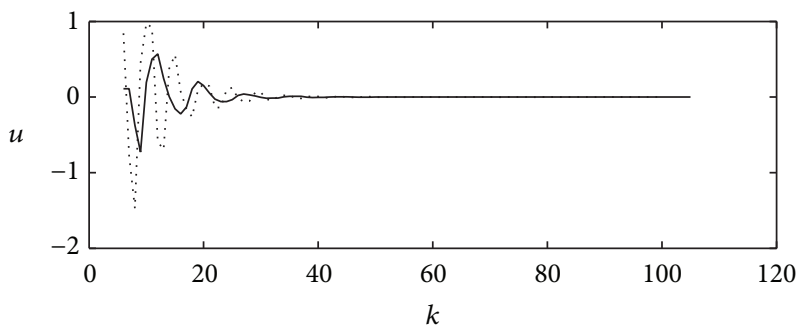

- RMPC-SC

..... RMPC

FIgURE 7: RMPC-SC $\times$ RMPC with Plant (38).

chosen before the comparison, in order to achieve the widest robustness. Given a pair of coefficients $(a, b)$, the stabilization was declared when the mean of difference $(y(k)-w(k))$ was lower than 0.01 , to $N / 2 \leq k \leq N$, for $N=200$.

Figure 11 shows the performance of the methods RGPC and RMPC-SC for controlling the plant (36) associated
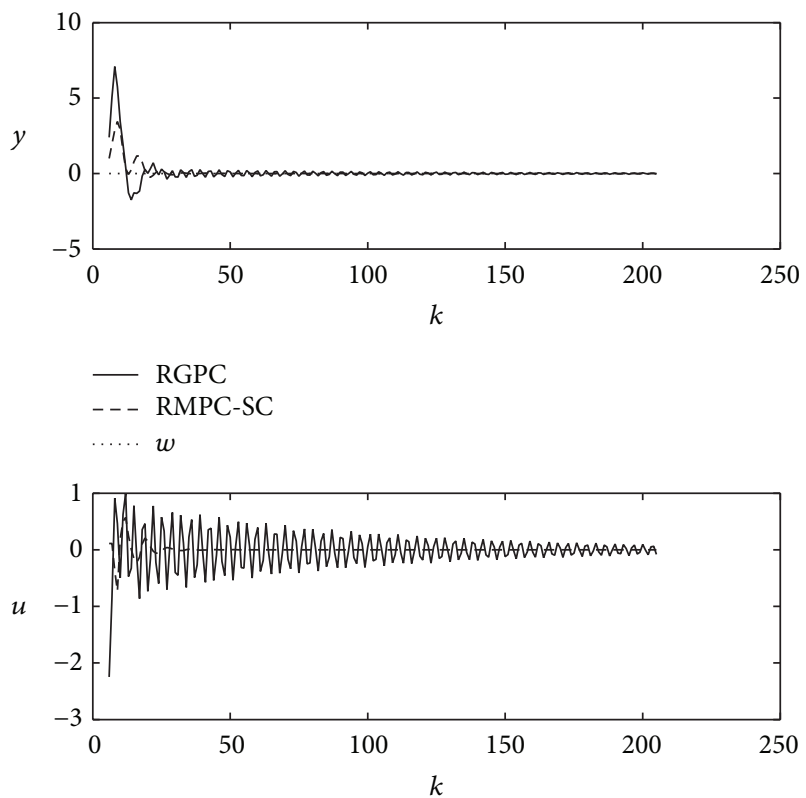

- RGPC
--- RMPC-SC

Figure 8: RGPC $\times$ RMPC-SC with Plant (38).
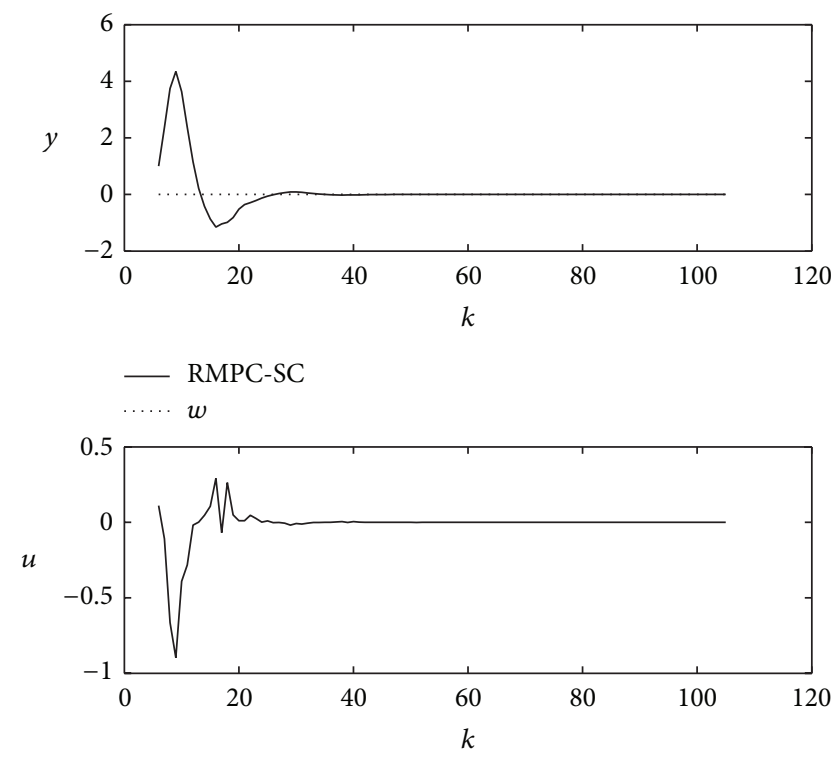

- RMPC-SC

FIgURE 9: RMPC-SC with Plant (39).

with each pair $(a, b)$, with nominal model corresponding to $(a, b)=(0.85,1.0)$. The RMPC-SC achieved stabilization for $35 / 36$ of the problem instances, while the RGPC achieved stabilization for only 19/36 of the problem instances.

The results displayed in Figure 11 reveal an interesting pattern: RMPC-SC leads to larger intervals of robustness for uncertainties in the parameters $(a, b)$ than RGPC. This is expected, since the kind of robustness provided by RGPC 


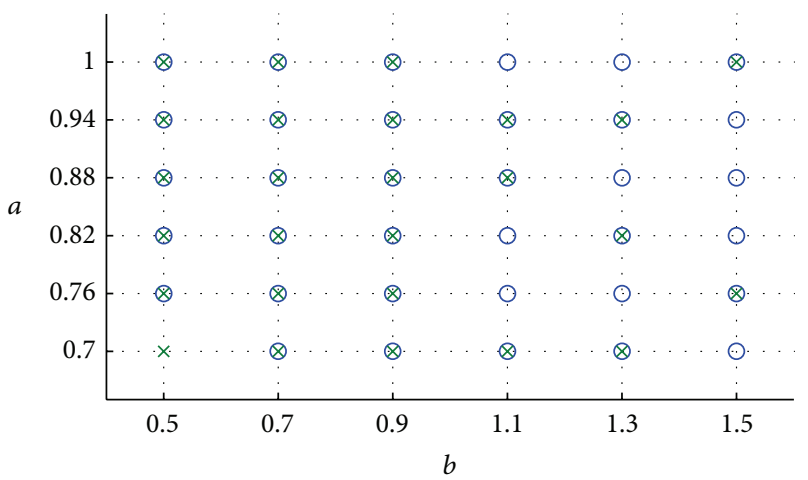

FIGURE 10: RMPC-SC $\times$ RMPC with Different instances of plant (36). O: satisfactory performance of RMPC-SC, $\times$ : satisfactory performance of RMPC.

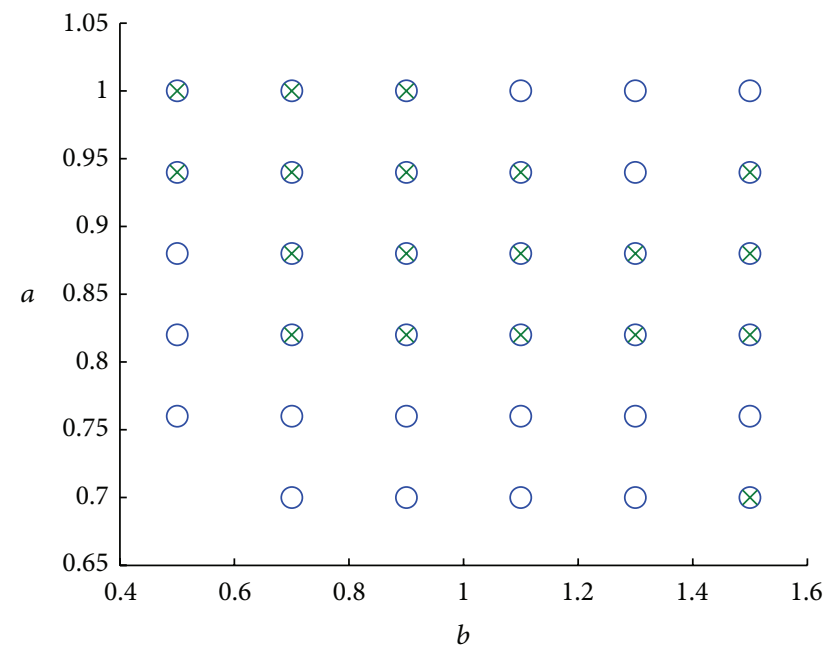

FIGURE 11: RMPC-SC $\times$ RGPC with Plant (36) associated with each pair $(a, b)$. RGPC with nominal model $(a, b)=(0.85,1.0)$. $\bigcirc$ : satisfactory performance of RMPC-SC, $\times$ : satisfactory performance of RGPC.

is suitable for norm-bounded uncertainties (in an infinitedimensional space). As RMPC-SC is specifically designed for providing robustness against parametric uncertainties in the $(a, b)$ directions, RMPC-SC is expected to be less conservative for those uncertainties, which is confirmed by the obtained results.

\section{Conclusions}

The issue of guaranteeing robust stability of box-bounded uncertain systems controlled by MPC strategies was dealt with in this work using a "first-principle" approach, based on a sufficient condition for the convergence of sequences which gives rise to a set of constraints on the control inputs. The proposed approach can be used jointly with most of the usual MPC design strategies, as long as it is implemented by the simple introduction of additional constraints in the control inputs. The usefulness of the proposed technique was illustrated in the examples, which showed a better robustness performance than other techniques, in the case of boxbounded uncertainties.

Two important limitations of the proposed technique should be mentioned: its high computational cost and its current availability only for SISO systems. The authors are currently studying alternatives for solving those issues.

\section{Conflict of Interests}

The authors declare that there is no conflict of interests regarding the publication of this paper.

\section{Acknowledgments}

This work was supported by the Brazilian agencies CAPES, CNPq, and FAPEMIG.

\section{References}

[1] E. Camacho and C. Bordons, Model Predictive Control, Springer, 2nd edition, 2004.

[2] C. E. Garcia, D. M. Prett, and M. Morari, "Model predictive control: theory and practice-a survey," Automatica, vol. 25, no. 3, pp. 335-348, 1989.

[3] J. M. Maciejowski, Predictive Control with Constraints, PrenticeHall, London, UK, 2002.

[4] K. S. Holkar and L. M. Waghmare, "An overview of model predictive control," International Journal of Control and Automation, vol. 3, no. 4, pp. 47-64, 2010.

[5] D. Q. Mayne, "Model predictive control: recent developments and future promise," Automatica, vol. 50, no. 12, pp. 2967-2986, 2014.

[6] J. A. Primbs and V. Nevistic, "A framework for robustness analysis of constrained finite receding horizon control," IEEE Transactions on Automatic Control, vol. 45, no. 10, pp. 1828$1838,2000$.

[7] H. K. Khalil, Nonlinear Systems, Prentice-Hall, 2002.

[8] J. B. Rawlings and D. Q. Mayne, Model Predictive Control: Theory and Design, Nob Hill, 2009.

[9] D. L. Marruedo, T. Álamo, and E. F. Camacho, "Input-to-state stable MPC for constrained discrete-time nonlinear systems with bounded additive uncertainties," in Proceedings of the 41st IEEE Conference on Decision and Control, vol. 4, pp. 4619-4624, IEEE, Las Vegas, Nev, USA, December 2002.

[10] P. O. Scokaert and D. Q. Mayne, "Min-max feedback model predictive control for constrained linear systems," IEEE Transactions on Automatic Control, vol. 43, no. 8, pp. 1136-1142, 1998.

[11] E. C. Kerrigan and J. M. Maciejowski, "Feedback min-max model predictive control using a single linear program: robust stability and the explicit solution," International Journal of Robust and Nonlinear Control, vol. 14, no. 4, pp. 395-413, 2004.

[12] D. Limon, T. Alamo, F. Salas, and E. F. Camacho, "On the stability of constrained MPC without terminal constraint," IEEE Transactions on Automatic Control, vol. 51, no. 5, pp. 832-836, 2006.

[13] D. R. Ramírez and E. F. Camacho, "On the piecewise linear nature of constrained min-max model predictive control with bounded uncertainties," in Proceedings of the American Control Conference, pp. 2453-2458, Denver, Colo, USA, June 2003. 
[14] M. Lazar, D. Muñoz de la Peña, W. P. Heemels, and T. Alamo, "On input-to-state stability of min-max nonlinear model predictive control," Systems \& Control Letters, vol. 57, no. 1, pp. 3948,2008

[15] G. Pin, D. M. Raimondo, L. Magni, and T. Parisini, "Robust model predictive control of nonlinear systems with bounded and state-dependent uncertainties," IEEE Transactions on Automatic Control, vol. 54, no. 7, pp. 1681-1687, 2009.

[16] L. Chisci, J. A. Rossiter, and G. Zappa, "Systems with persistent disturbances: predictive control with restricted constraints," Automatica, vol. 37, no. 7, pp. 1019-1028, 2001.

[17] D. Q. Mayne and W. Langson, "Robustifying model predictive control of constrained linear systems," Electronics Letters, vol. 37, no. 23, pp. 1422-1423, 2001.

[18] D. Q. Mayne, M. M. Seron, and S. V. Raković, "Robust model predictive control of constrained linear systems with bounded disturbances," Automatica, vol. 41, no. 2, pp. 219-224, 2005.

[19] J. E. Normey-Rico and E. F. Camacho, "Robustness effects of a prefilter in a Smith predictor-based generalised predictive controller," IEE Proceedings-Control Theory and Applications, vol. 146, no. 2, pp. 179-185, 1999.

[20] J. E. Normey-Rico and E. F. Camacho, "Multivariable generalised predictive controller based on the Smith predictor," IEE Proceedings-Control Theory and Applications, vol. 147, no. 5, pp. 538-546, 2000.

[21] T. L. M. Santos, D. Limon, J. E. Normey-Rico, and T. Alamo, "On the explicit dead-time compensation for robust model predictive control," Journal of Process Control, vol. 22, no. 1, pp. 236-246, 2012.

[22] C. Løvaas, M. M. Seron, and G. C. Goodwin, "Robust outputfeedback model predictive control for systems with unstructured uncertainty," Automatica, vol. 44, no. 8, pp. 1933-1943, 2008.

[23] P. Falugi and D. Q. Mayne, "Getting robustness against unstructured uncertainty: a tube-based MPC approach," IEEE Transactions on Automatic Control, vol. 59, no. 5, pp. 1290-1295, 2014.

[24] S. S. Ge, G. Y. Li, and T. H. Lee, "Adaptive NN control for a class of strict-feedback discrete-time nonlinear systems," Automatica, vol. 39, no. 5, pp. 807-819, 2003.

[25] C. Yang, S. S. Ge, C. Xiang, T. Chai, and T. H. Lee, "Output feedback NN control for two classes of discrete-time systems with unknown control directions in a unified approach," IEEE Transactions on Neural Networks, vol. 19, no. 11, pp. 1873-1886, 2008.

[26] Y.-J. Liu and S. Tong, "Adaptive neural network tracking control of uncertain nonlinear discrete-time systems with nonaffine dead-zone input," IEEE Transactions on Cybernetics, vol. 45, no. 3, pp. 497-505, 2015.

[27] S. S. Ge, C. Yang, and T. H. Lee, "Adaptive predictive control using neural network for a class of pure-feedback systems in discrete time," IEEE Transactions on Neural Networks, vol. 19, no. 9, pp. 1599-1614, 2008.

[28] Y.-J. Liu, L. Tang, S. Tong, and C. L. P. Chen, "Adaptive NN controller design for a class of nonlinear MIMO discrete-time systems," IEEE Transactions on Neural Networks and Learning Systems, vol. 26, no. 5, pp. 1007-1018, 2014.

[29] H. Du, G. L. Fan, and J. Q. Yi, "Autonomous takeoff control system design for unmanned seaplanes," Ocean Engineering, vol. 85, pp. 21-31, 2014.

[30] Y. K. Ho, H. K. Yeoh, and F. S. Mjalli, "Generalized predictive control algorithm with real-time simultaneous modeling and tuning," Industrial and Engineering Chemistry Research, vol. 53, no. 22, pp. 9411-9426, 2014.

[31] G. Y. Tang, D. M. Huang, and Z. Y. Deng, "T-S fuzzy model based generalized predictive control of vehicle yaw stability," Kybernetes, vol. 41, no. 9, pp. 1261-1268, 2012.

[32] X. J. Mao, D. Wang, W. Y. Xiao, Z. Y. Liu, J. X. Wang, and H. B. Tang, "Lean limit and emissions improvement for a sparkignited natural gas engine using a generalized predictive control (GPC)-based air/fuel ratio controller," Energy and Fuels, vol. 23, no. 12, pp. 6026-6032, 2009.

[33] H. Hapoglu, S. Karacan, Z. S. Erten Koca, and M. Alpbaz, "Parametric and nonparametric model based control of a packed distillation column," Chemical Engineering and Processing, vol. 40, no. 6, pp. 537-544, 2001.

[34] M. Mahfouf, M. F. Abbod, and D. A. Linkens, "Online elicitation of Mamdani-type fuzzy rules via TSK-based generalized predictive control," IEEE Transactions on Systems, Man, and Cybernetics Part B: Cybernetics, vol. 33, no. 3, pp. 465-475, 2003.

[35] A. Sabanovic and O. Ohnishi, Motion Control Systems, IEEE Press, 2011.

[36] A. M. Barbosa, R. H. C. Takahashi, and L. A. Aguirre, "Equivalence of non-linear model structures based on Pareto uncertainty," IET Control Theory \& Applications, vol. 9, no. 16, pp. 2423-2429, 2015.

[37] D. W. Clarke, C. Mohtadi, and P. S. Tuffs, "Generalized predictive control. Part I. The basic algorithm," Automatica, vol. 23, no. 2, pp. 137-148, 1987.

[38] H. Kwakernaak and R. Sivan, Linear Optimal Control Systems, Wiley-Interscience, New York, NY, USA, 1972.

[39] M. V. Kothare, V. Balakrishnan, and M. Morari, "Robust constrained model predictive control using linear matrix inequalities," Automatica, vol. 32, no. 10, pp. 1361-1379, 1996. 


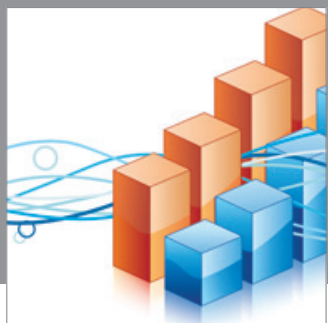

Advances in

Operations Research

mansans

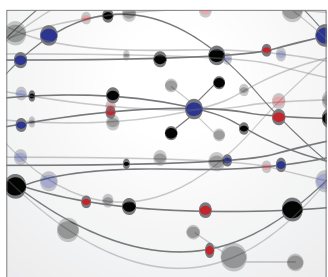

The Scientific World Journal
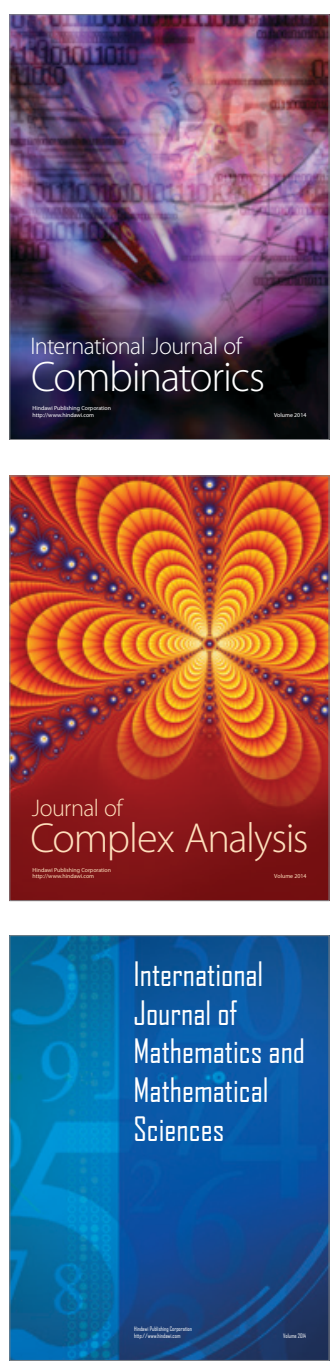
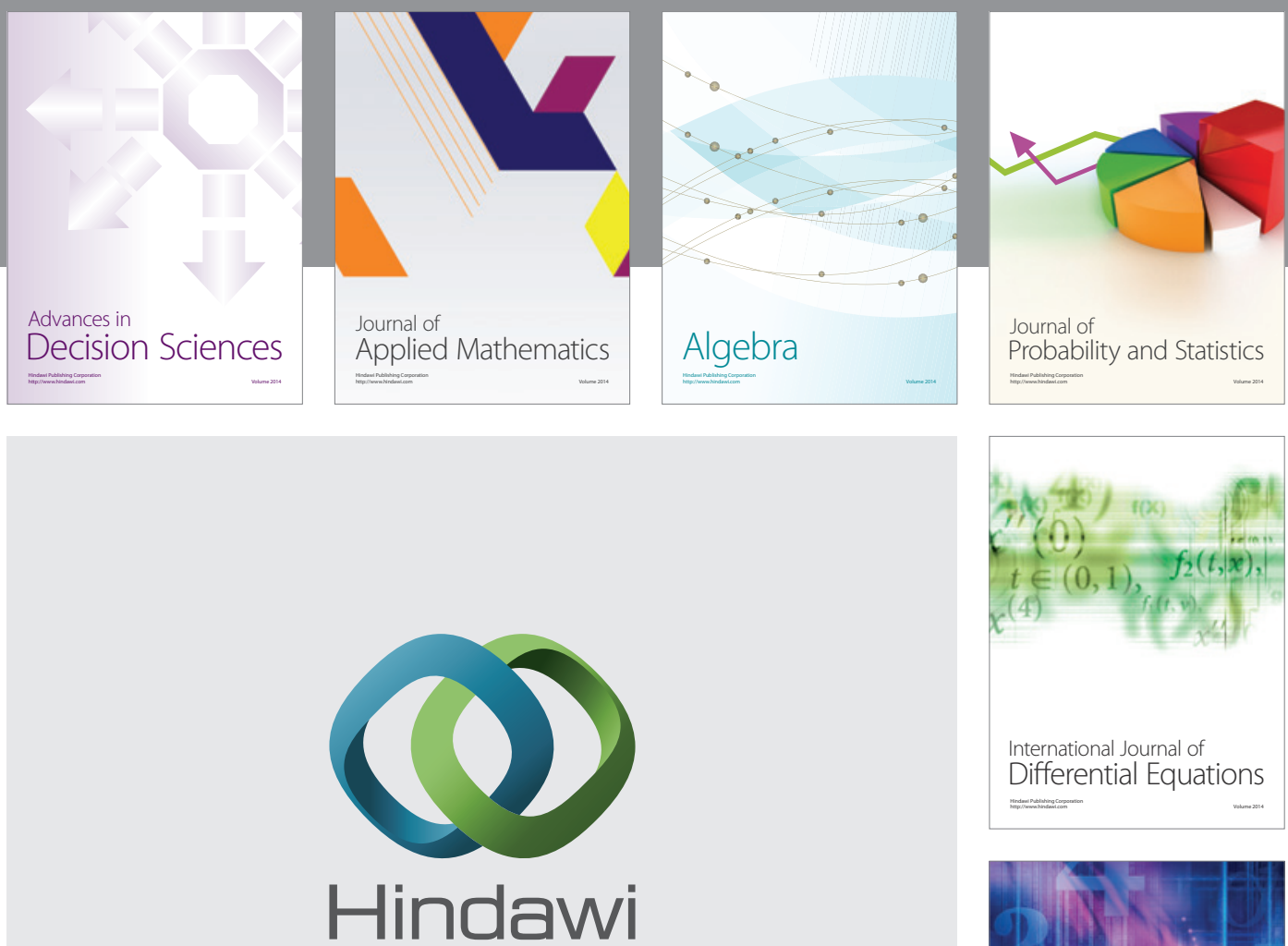

Submit your manuscripts at http://www.hindawi.com
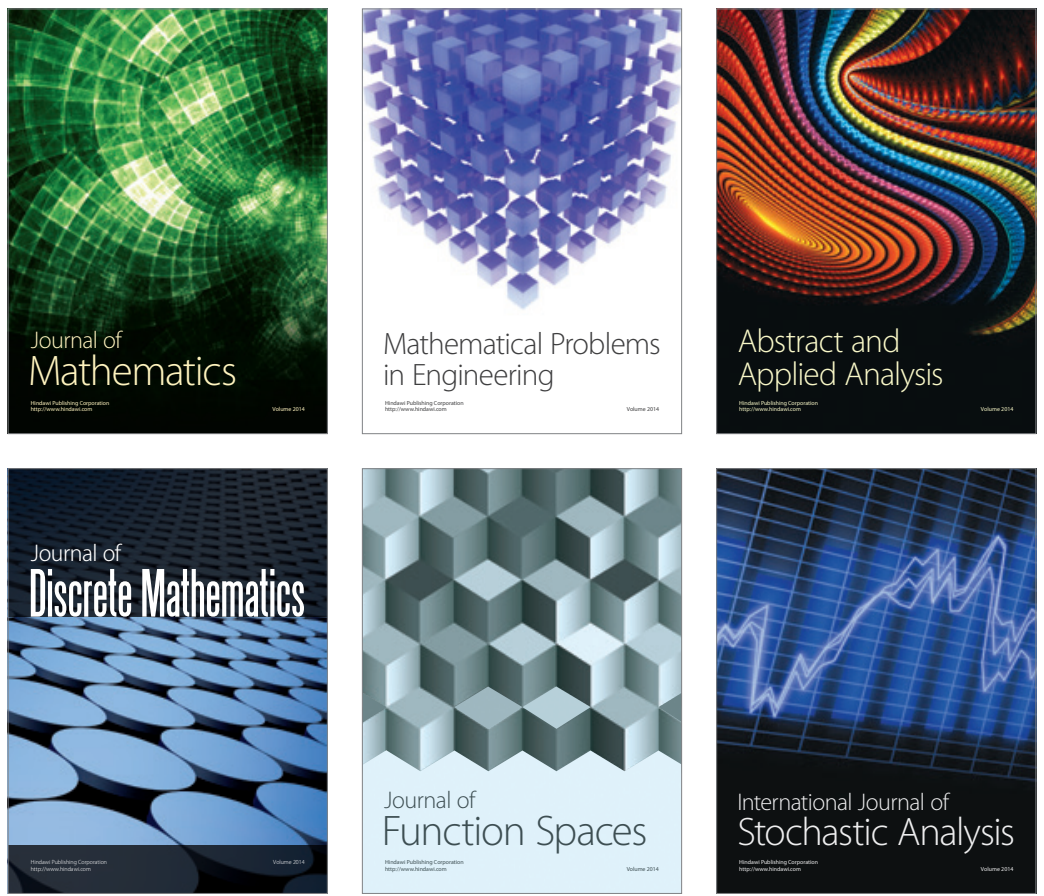

Journal of

Function Spaces

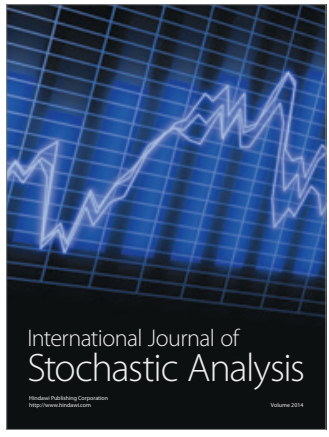

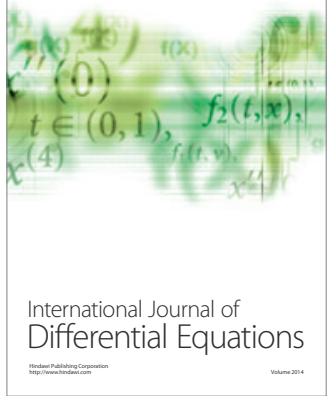
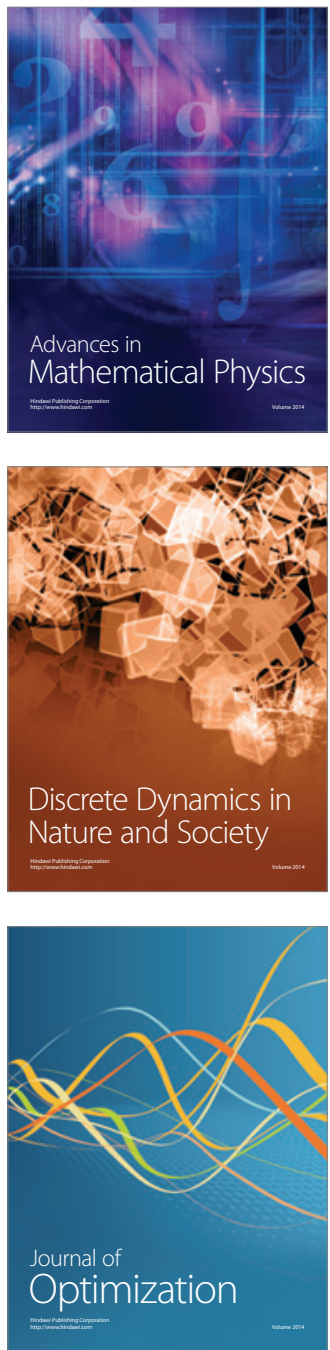